\title{
Avaliação da qualidade do serviço odontológico prestado pela Faculdade de Odontologia da Universidade de Itaúna, MG, Brasil: visão do usuário, 2014
}

\author{
Assessment of dental service quality provided by the School of Dentistry, \\ University of Itaúna, MG, Brazil: user view, 2014
}

Rayanne Cristhina Borges*

Thaína Aparecida Chaves Otoni*

Regina Coeli Cançado Peixoto Pires ${ }^{* *}$

\section{Resumo}

Introdução: identificar o padrão socioeconômico de usuários de serviços públicos é de suma importância para planejar procedimentos a serem realizados nas clínicas de instituições de ensino superior além de funcionar como aprendizado na construção do plano de tratamento a ser proposto pelo aluno para cada paciente. Objetivo: o objetivo deste estudo é avaliar o perfil sociodemográfico e o grau de satisfação dos pacientes atendidos nas clínicas da Faculdade de Odontologia da Universidade de Itaúna, MG, e os serviços prestados pelos funcionários, alunos e professores. Materiais e método: foi utilizado um questionário autoaplicável elaborado especificamente para o estudo, que foi respondido sem intervenção dos pesquisadores. Os participantes foram pacientes que frequentaram as clínicas da Faculdade de Odontologia, de segunda a sexta-feira, nos turnos manhã e tarde, e sábado pela manhã. Após cálculo amostral, a população total do estudo foi constituída por 227 usuários, sendo estratificados proporcionalmente pelo dia da semana de atendimento. Resultados: os resultados demonstraram que 159 (70,0\%) usuários eram do gênero feminino e apresentavam baixa escolaridade, e 138 (60,8\%) tinham renda mensal de dois a três salários mínimos. Os dados mostraram que 101 indivíduos $(32,8 \%)$ buscaram atendimento odontológico na faculdade pela impossibilidade financeira de procurar serviço particular, 190 (83,7\%) confiavam nos procedimentos realizados pelos alunos. A faculdade teve uma avaliação positiva por 205 (90,3\%) usuários do serviço. Conclusão: os pacientes mostraram-se satisfeitos com os atendimentos realizados na Faculdade de Odontologia, refletindo o bom desempenho de alunos, professores e funcionários. Essa avaliação é importante por fornecer subsídios para o planejamento de medidas, visando a melhorias nos serviços prestados.

Palavras-chave: Avaliação. Odontologia. Pacientes.

\section{Introdução}

Apesar de o Sistema Único de Saúde ter sido legalmente estruturado há quase duas décadas ${ }^{1}$, o Suplemento de Saúde da Pesquisa Nacional por Amostra de Domicílios, de 2008, mostra que 11,7\% dos brasileiros nunca receberam atendimento odontológico ${ }^{2}$, demonstrando que ainda há uma grande parte da população sem acesso a esses serviços. Nesse contexto, as faculdades de odontologia ganham especial destaque no que tange à melhora ao acesso da população em relação à saúde bucal ${ }^{1}$ como mais uma oportunidade de atendimento. 
Uma instituição de ensino odontológico tem de extrapolar a formação técnica de profissionais, atuando direta e indiretamente sobre a realidade social da área em que está inserida ${ }^{3}$. Para tanto, torna-se indispensável conhecer as condições de vida do paciente para realizar um atendimento integral ${ }^{4}$.

Estudos sobre a satisfação dos usuários dos serviços de saúde são importantes, pois avaliam a qualidade desses serviços ${ }^{5}$ por meio da percepção dos pacientes, mostrando a realidade vivida por eles, e constitui-se em uma importante ferramenta de auxílio na implementação de mudanças e melhorias nessas instituições.

Quanto mais o cirurgião-dentista busca compreender as pessoas que o procuram na busca de um auxílio técnico, mais objetivo e humanitário poderá tornar-se o tratamento. Portanto, uma boa relação profissional-paciente deve se constituir em uma estratégia para conduzir comportamentos adequados de ambos os indivíduos em interação ${ }^{6}$.

Um processo avaliativo de serviços de saúde, contudo, não seria considerado um fim em si mesmo, mas, sim, um momento em que o julgamento explícito é elaborado. A partir disso desencadear-se-ia um movimento de transformação na busca da melhoria da qualidade do atendimento. A verificação da satisfação dos pacientes é o melhor indicativo para avaliar qual padrão de atendimento do serviço de saúde odontológica está sendo oferecido ao usuário ${ }^{4}$.

Assim, os estudos sobre satisfação do usuário são importantes, porque podem contribuir para o planejamento de medidas visando à superação das limitações detectadas com base nas informações adquiridas ${ }^{1}$. Diante do exposto, este estudo tem por objetivo analisar a satisfação e o perfil sociodemográfico dos usuários das clínicas da Faculdade de Odontologia da Universidade de Itaúna (Foui), a partir dos serviços prestados por essa instituição.

\section{Materiais e método}

Este estudo foi aprovado pelo Comitê de Ética em Pesquisa da Universidade de Itaúna (CAAE 31145014.0.0000.5144), e os indivíduos que concordaram em participar da pesquisa assinaram o termo de consentimento livre e esclarecido. Trata-se de um estudo transversal, descritivo. A população total estimada foi de 520 pacientes, referentes à contagem mensal de todos os prontuários de usuários em atendimento na Faculdade de Odontologia da Universidade de Itaúna, Minas Gerais, nos meses de agosto a novembro de 2013. Após a definição do plano amostral, o resultado da amostra final contou com 227 pacientes. Eles foram selecionados por meio de uma amostragem aleatória estratificada proporcional aos dias da semana, correspondente ao atendimento.

Os dados foram coletados por meio de um questionário autoaplicável, elaborado especificamente para o estudo, que foi entregue aos 227 pesquisados por ordem de chegada, e respondido pelos pacientes que aguardavam atendimento na sala de espera das clínicas da Faculdade de Odontologia, de segunda a sexta-feira, nos períodos da manhã e tarde, e sábado pela manhã. No caso de haver recusa para preenchimento do questionário, ele era passado para o próximo paciente.

Os dados foram tabulados e analisados utilizando-se ferramentas estatísticas do software SPSS for Windows ${ }^{\circledR}$, versão 17.0, no qual foram geradas tabelas de frequência, análise descritiva e associações de variáveis para caracterizar cada resposta dentro da população-alvo.

\section{Resulltados}

A Tabela 1 descreve o perfil sociodemográfico dos 227 pacientes entrevistados. Verificou-se que 64 pacientes $(28,0 \%)$ eram adultos, com idade de 41 a 60 anos, e 159 (70,0\%) eram do gênero feminino, sendo que $119(52,4 \%)$ tinham somente o ensino fundamental e $138(60,8 \%)$ tinham uma renda mensal de dois a três salários mínimos. Para a avaliação da renda familiar, considerou-se o salário mínimo de referência no ano de 2014, com valor de $\mathrm{R} \$ 724,00$.

Tabela 1 -Distribuição dos usuários entrevistados de acordo com o perfil sociodemográfico

\begin{tabular}{|c|c|c|}
\hline Variáveis & № & $\%$ \\
\hline \multicolumn{3}{|l|}{ Idade (anos) } \\
\hline $10-20$ & 9 & 4,0 \\
\hline $21-40$ & 56 & 24,6 \\
\hline $41-60$ & 64 & 28,0 \\
\hline $61-80$ & 21 & 9,2 \\
\hline Não respondeu & 77 & 33,9 \\
\hline Total & 227 & 100,0 \\
\hline \multicolumn{3}{|l|}{ Sexo } \\
\hline Masculino & 62 & 27,3 \\
\hline Feminino & 159 & 70,0 \\
\hline Não respondeu & 6 & 2,6 \\
\hline Total & 227 & 100,0 \\
\hline \multicolumn{3}{|l|}{ Escolaridade } \\
\hline Não estudou & 9 & 4,0 \\
\hline Ensino fundamental & 119 & 52,4 \\
\hline Ensino médio incompleto & 22 & 9,7 \\
\hline Ensino médio completo & 53 & 23,3 \\
\hline Ensino superior incompleto & 12 & 5,2 \\
\hline Ensino superior completo & 10 & 4.4 \\
\hline Não respondeu & 2 & 0,9 \\
\hline Total & 227 & 100,0 \\
\hline \multicolumn{3}{|l|}{ Renda familiar } \\
\hline Até R\$ 720,00 & 25 & 11,0 \\
\hline De $R \$ 1.440,00$ a $R \$ 2.160,00$ & 138 & 60,8 \\
\hline De $R \$ 2.160,00$ a $R \$ 7.200,00$ & 22 & 9,7 \\
\hline Acima de R\$ 7.200,00 & 3 & 1,3 \\
\hline Nenhuma renda & 28 & 12,3 \\
\hline Não respondeu & 11 & 4,8 \\
\hline Total & 227 & 100,0 \\
\hline
\end{tabular}

Fonte: todas as tabelas são de elaboração dos autores com base nos dados da pesquisa. 
Observou-se que, dentre as razões atribuídas pelos entrevistados para a procura pelo atendimento odontológico nas clínicas da faculdade, a mais citada foi a falta de condições de pagar o tratamento em outro lugar, seguida da qualificação do serviço prestado pela Foui, conforme Tabela 2.

Tabela 2 - Distribuição dos usuários entrevistados de acordo com o motivo que os levou a procurar atendimento na clínica da Foui*

\begin{tabular}{l|r|r}
\multicolumn{1}{c|}{ Variáveis } & \multicolumn{1}{c|}{ № } & \multicolumn{1}{c}{$\%$} \\
\hline Indicação de amigo & 64 & 20,7 \\
Ser conhecido do aluno & 13 & 4,2 \\
Não concluiu o tratamento no ano & 22 & 7,1 \\
anterior & 101 & 32,8 \\
Não poder pagar & 34 & 11,0 \\
Indicação de dentista particular/ & 74 & 24,0 \\
posto de saúde & 308 & 100,0 \\
Qualidade do serviço &
\end{tabular}

Os resultados indicaram que os alunos apresentavam confiança e segurança para realizar os procedimentos, segundo a opinião dos usuários (Tabela 3).

Tabela 3 -Distribuição dos usuários entrevistados de acordo com avaliação sobre a segurança dos alunos na realização de procedimentos

\begin{tabular}{l|r|r}
\hline \multicolumn{1}{c|}{ Variáveis } & \multicolumn{1}{c|}{ № } & \multicolumn{1}{c}{$\%$} \\
\hline Não & 1 & 0,4 \\
Sim & 190 & 83,7 \\
Em parte & 18 & 7,9 \\
Não respondeu & 18 & 7,9 \\
Total & 227 & 100,0 \\
\hline
\end{tabular}

A Tabela 4 apresenta a associação entre as explicações fornecidas pelos alunos em relação aos procedimentos a serem realizados e ao entendimento do usuário.

Tabela 4 - Distribuição das respostas dos usuários segundo explicação dos alunos sobre os procedimentos realizados e o entendimento

\begin{tabular}{|c|c|c|c|c|c|c|}
\hline & \multicolumn{4}{|c|}{$\begin{array}{l}\text { As explicações } \\
\text { foram claras }\end{array}$} & \multirow{2}{*}{ Total } \\
\hline & & NR & Não & Sim & $\begin{array}{l}\text { Em } \\
\text { parte }\end{array}$ & \\
\hline \multirow{4}{*}{$\begin{array}{l}\text { Aluno } \\
\text { explicou o } \\
\text { procedimento }\end{array}$} & Não & 0 & 2 & 0 & 2 & 4 \\
\hline & Sim & 2 & 0 & 180 & 11 & 193 \\
\hline & Em parte & 0 & 0 & 6 & 7 & 13 \\
\hline & NR* & 13 & 0 & 4 & 0 & 17 \\
\hline \multicolumn{2}{|l|}{ Total } & 15 & 2 & 190 & 20 & 227 \\
\hline
\end{tabular}

${ }^{*} \mathrm{NR}=$ Não respondeu.

Ao solicitar que os participantes avaliassem o serviço prestado por professores e funcionários, ambos foram classificados como ótimos (Tabela 5).
Tabela 5 - Avaliação dos usuários dos serviços e atendimentos prestados pelos professores e funcionários da Foui

\begin{tabular}{l|r|r|r|r}
\hline \multirow{2}{*}{ Variáveis } & \multicolumn{4}{|c}{ № (\%) } \\
\cline { 2 - 5 } & \multicolumn{2}{|c}{ Professor } & \multicolumn{2}{c}{ Funcionário } \\
\hline Ótimo & 159 & 70,0 & 141 & 62,1 \\
Bom & 51 & 22,5 & 77 & 33,9 \\
Regular & 11 & 4,8 & 2 & 0,9 \\
Péssimo & 6 & 2,6 & 7 & 3,1 \\
Total & 227 & 100,0 & 227 & 100,0 \\
\hline
\end{tabular}

Quanto ao tratamento realizado, $205(90,3 \%)$ dos usuários manifestaram-se como satisfeitos ou muito satisfeitos (Tabela 6).

Tabela 6 -Distribuição dos usuários quanto à satisfação com o tratamento realizado pela Foui

\begin{tabular}{l|r|r}
\multicolumn{1}{c|}{ Variáveis } & \multicolumn{1}{c|}{ № } & \multicolumn{1}{c}{$\%$} \\
\hline Muito satisfeito & 118 & 52,0 \\
Satisfeito & 87 & 38,3 \\
Um pouco satisfeito & 17 & 7,5 \\
Insatisfeito & 5 & 2,2 \\
Total & 227 & 100,0 \\
\hline
\end{tabular}

Na questão aberta, que solicitava sugestões para a melhoria do atendimento da clínica, observou-se que as respostas mais frequentes foram: "reduzir o tempo de espera no banco da sala de espera" e "ser atendido mais vezes durante a semana", conforme descrito na Tabela 7.

Tabela 7 - Sugestões apontadas pelos pacientes para a melhoria do atendimento da Foui

\begin{tabular}{l|r|r}
\multicolumn{1}{c|}{ Variáveis } & \multicolumn{1}{c|}{ № } & \multicolumn{1}{c}{$\%$} \\
\hline Reduzir o tempo de espera & 82 & 34,0 \\
Ser atendido mais vezes por semana & 80 & 33,2 \\
Reduzir o tempo de atendimento & 22 & 9,1 \\
Sem sugestão & 57 & 23,6 \\
Total & 241 & 100,0 \\
\hline
\end{tabular}

\section{Discussão}

Identificar o padrão socioeconômico de usuários de serviços públicos é de suma importância para o planejamento dos procedimentos a serem realizados nas clínicas de instituições de ensino superior, além de funcionar como aprendizado na construção do plano de tratamento a ser proposto pelo aluno para cada paciente ${ }^{5}$.

Observou-se que adultos de 41 a 60 anos foram os usuários que mais procuraram atendimento nas clínicas da Faculdade de Odontologia. Os dois últimos levantamentos epidemiológicos para doenças bucais realizados no Brasil, SB Brasil 2003 e 2010, demonstraram a grande necessidade de atendimento odontológico para essa faixa da população. 
Os resultados desses levantamentos descreveram que há uma tendência de crescimento na prevalência da cárie dentária em função da idade, e que em adultos e idosos, em geral, a redução no ataque de cárie é menos significativa, tendo em conta o caráter cumulativo das sequelas da doença. Analisando os resultados para o grupo de 35 a 44 anos, observa-se que o índice de dentes cariados, perdidos e obturados (CPOD) caiu de 20,1, em 2003, para 16,7, em 2010, mantendo-se ainda elevado mesmo com esse declínio. Entre os idosos, o CPOD praticamente não se alterou, mantendo-se em torno de 27, com a maioria correspondendo ao componente extraído, refletindo a necessidade de algum tipo de prótese ${ }^{7,8}$. Em relação às condições periodontais avaliadas pelo Índice Periodontal Comunitário, em termos populacionais, esses problemas aumentam, de modo geral, com a idade ${ }^{8}$.

A análise desses dados pode justificar a busca por atendimento na Faculdade de Odontologia para essa faixa etária, por necessidade de tratamento especializado, como procedimentos restauradores, reabilitadores e periodontais, pois são mais facilmente disponibilizados do que nos centros de atendimento de programas de saúde bucal.

Observou-se também que as mulheres foram maioria na procura por atendimento. Uma possível explicação para esses resultados seria o horário de funcionamento das clínicas da faculdade, diurno, e porque as mulheres têm mais flexibilidade de horários e se preocupem mais com a aparência. Essas observações também foram relatadas em outros estudos ${ }^{5,9-13}$, que apresentaram percentagens elevadas referentes a participantes do gênero feminino, entre $136(68 \%)$ e $48(90,6 \%)$.

Oliveira et al. ${ }^{10}$ (2012) e Fernando et al. ${ }^{11}$ (2008) relataram que a renda da maioria dos participantes esteve, em geral, abaixo de três salários mínimos, a mesma condição foi observada neste estudo, em $138(60,8 \%)$ dos usuários entrevistados, o que pode estar relacionado com o baixo nível de escolaridade citado, pois $119(52,4 \%)$ usuários têm somente o ensino fundamental.

Os motivos verificados para a procura do tratamento na faculdade foram a impossibilidade financeira de procurar serviço particular e a qualidade do tratamento prestado pela instituição, o que reflete a importância da universidade para a cidade e a população da região na qual está inserida.

Os usuários entrevistados afirmaram que os alunos demonstraram confiança e segurança na realização do atendimento clínico, fornecendo explicações claras sobre os procedimentos realizados. Verificou-se, portanto, um alto grau de satisfação com o atendimento prestado pelos alunos, professores e funcionários nas clínicas da Foui, pois 205 (90,3\%) pacientes disseram estar satisfeitos ou muito satisfeitos.

O estudo de Watanabe et al. ${ }^{14}$ (1997) apontou que tal classificação se justifica também pela influ- ência do fator socioeconômico, já que o baixo custo dos procedimentos faz com que os usuários apresentem baixa expectativa em relação ao tratamento e sejam mais tolerantes. Por ser um estudo datado de 1997, quando o acesso ao tratamento público odontológico era diferente do ofertado atualmente e a população não disponibilizava tão facilmente de meios de informação, acredita-se que esse comentário pode não ser uma justificativa para a satisfação dos usuários das clínicas da Foui.

Já segundo Mialhe et al. ${ }^{1}$ (2008), três fatores pesam para que seja mantida essa situação, ou seja, a expectativa do usuário de que seu tratamento esteja sendo supervisionado por um profissional muito bem titulado, a falta de condições para contratar um serviço particular e a inexistência da oferta desse serviço com a mesma qualidade no âmbito público. Dessa forma, nada resta além de aceitar de forma submissa as condições que as instituições de ensino lhes oferecem.

Como sugestões para melhoria no atendimento, as mais citadas foram reduzir o tempo de espera e aumentar a frequência semanal das consultas, o que demonstra o desejo de um atendimento mais rápido, reduzindo o tempo de conclusão do tratamento, que é a maior dificuldade no atendimento em instituições de ensino. Isso ocorre devido às interrupções no tratamento devido a férias, provas, pouca habilidade do aluno e o tempo gasto de explicação pelo professor daquele procedimento a ser realizado.

\section{Conclusão}

De acordo com a pesquisa, podemos concluir que há grande satisfação dos usuários quanto à qualidade do serviço odontológico prestado pelo Curso de Odontologia da Universidade de Itaúna, apesar de essa avaliação estar ligada ao fator socioeconômico dos pacientes.

O processo de atendimento precisa ser aperfeiçoado, principalmente devido à demora do tratamento odontológico, pois como o objetivo principal é a formação de profissionais altamente qualificados, cada procedimento é acompanhado e avaliado pelo professor conforme o aprendizado de cada aluno. Como proposta de solução, sugere-se a explicar detalhadamente as normas de atendimento ao paciente ${ }^{15}$ em relação a essa observação.

A pesquisa demonstrou também a importância da avaliação da qualidade dos serviços prestados pelas clínicas das Faculdades de Odontologia, sob a ótica dos pacientes, fornecendo subsídios para aqueles que as gerenciam e para a equipe que presta os cuidados, possibilitando superação das limitações detectadas. 


\section{Agradecimento}

Esta pesquisa teve apoio financeiro da Fundação de Amparo à Pesquisa do Estado de Minas Gerais, por meio do Programa de Apoio, Fomento e Acompanhamento de Pesquisa da Universidade de Itaúna.

\section{Abstract}

Introduction: Identifying the socioeconomic pattern of public service users is essential to plan the procedures to be performed at clinics of higher education institutions, and it helps to perfect the construction of the treatment plan to be proposed by the student to each patient. Objective: This study aimed to assess the sociodemographic profile and the level of satisfaction of patients treated at the Dental School clinics of the University of Itaúna, Minas Gerais, Brazil, according to services provided by employees, students, and teachers. Material and Methods: a self-administered questionnaire, specifically designed for the study, was applied and responded with no intervention from researchers. The participants were patients who attended the Dental School clinics from Monday to Friday in the morning and afternoon, and by Saturday morning. After sample calculation, the total population of the study consisted of 227 users, proportionally stratified by day of the week they were assisted. Results: The results showed that 159 $(70.0 \%)$ users were women with low level of education, and $138(60.8 \%)$ had a monthly income of 2-3 minimum wages. Data showed that 101 (32.8\%) users sought dental care at the University because of financial inability to seek private care, $190(83.7 \%)$ trusted the procedures performed by the students, so the Dentistry School had a positive assessment from 205 (90.3\%) users. Conclusion: Patients showed to be satisfied with the care provided at the Dental School, which reflects the good performance of students, professors, and staff, given such assessment is important to aid the planning of actions, aiming to improve services provided.

Keywords: Assessment. Dentistry. Patients.

\section{Referências}

1. Mialhe FL, Gonçalo C, Carvalho LMS. Avaliação dos usuários sobre a qualidade do serviço odontológico prestado por graduandos do curso de Odontologia da FOP/Unicamp. Rev Fac Odontol Univ Passo Fundo 2008; 13(1):19-24.

2. Instituto Brasileiro de Geografia e Estatística. Pesquisa nacional por amostra de domicílios (PNAD). Um panorama da saúde no Brasil: acesso e utilização dos serviços, condições de saúde e fatores de risco e proteção à saúde 2008. Rio de Janeiro: IBGE; 2010.

3. Almeida RVD, Gaião L, Padilha WWN. Avaliação do ensino odontológico em clínica integrada. Pesqu Bras Odontoped Clin Integr 2001; 1(2):29-35.

4. Nobre ES, Câmara GP, Silva KP, Nuto SAS. Avaliação da qualidade de serviço odontológico prestado por universidade privada: visão do usuário. Rev Bras Promoç Saúde 2005; 18(4):171-6
5. D’Ávila S, Souza LMM, Cavalcanti GMS, Lucas RSCC, Garcia AFG, Cavalcanti AL. Nível de satisfação e condição socioeconômica dos usuários das clínicas de odontologia da Universidade Estadual da Paraíba. Rev Bras Pesqui Saúde 2010; 12(2):39-45.

6. Garbin CAS, Mariano RQ, Machado TP, Garbin AJI. Estudo bioético das relações humanas no tratamento odontológico. Rev Fac Odontol Lins 2002; 14(1):54-9.

7. Projeto SB Brasil 2003: condições de saúde bucal da população brasileira 2002-2003: resultados principais / Ministério da Saúde, Secretaria de Atenção à Saúde, Departamento de Atenção Básica. Brasília: Ministério da Saúde; 2004.

8. SB Brasil 2010: Pesquisa Nacional de Saúde Bucal: resultados principais / Ministério da Saúde. Secretaria de Atenção à Saúde. Secretaria de Vigilância em Saúde. Brasília: Ministério da Saúde; 2012.

9. Bottan ER, Sperb RAL, Telles PS, Neto MU. Avaliação de serviços odontológicos: a visão dos pacientes. Rev Abeno 2006; 6(2):128-33.

10. Oliveira OR, Martins EP, Santana JLB, Bezerra SRS, Dourado AT. Avaliação dos usuários sobre a qualidade do atendimento odontológico prestado por alunos de odontologia. RFO UPF 2012; 17(3):319-25.

11. Fernandes SKS, Coutinho ACM, Pereira EL. Avaliação do perfil socioeconômico e nível de satisfação dos pacientes atendidos em clínica integrada odontológica universitária. Rev Bras Promoç Saúde 2008; 21(2):137-43.

12. Medeiros FA, Souza GCA, Barbosa AAA, Costa ICC. Acolhimento em uma unidade básica de saúde: a satisfação do usuário em foco. Rev Salud Publica 2010; 12(3):402-13.

13. Tiedmann CR, Linhares E, Silveira JLGC. Clínica integrada odontológica: perfil e expectativas dos usuários e alunos. Pesqu Bras Odontoped Clin Integr 2005; 5(1):53-8.

14. Watanabe MGC, Agostinho AM, Moreira A. Aspectos socioeconômicos dos pacientes atendidos nas clínicas da Faculdade de Odontologia de Ribeirão Preto-USP. Rev Fac Odontol Univ São Paulo 1997; 11(2):147-51.

15. Castro JDB, Silva VB. Satisfação no atendimento odontológico: um estudo na COE (clínica odontológica de ensino) de Anápolis - Unievangélica. Rev Administra-Ação 2008; 5:34-43.

Endereço para correspondência:

Rayanne Cristhina Borges

Rua Gonçalves da Guia, 124, apto. 103, Centro

35680-009 Itaúna-MG

Telefone: (37) 8825-3158

E-mail: nanaborges2006@hotmail.com

Recebido: 04/08/15. Aceito: 20/10/15. 